\title{
Mediterranean diet and other lifestyle factors in relation to 20-year all-cause mortality: a cohort study in an Italian population
}

\author{
Federica Prinelli ${ }^{1,2 *}$, Mary Yannakoulia ${ }^{3}$, Costas A. Anastasiou ${ }^{3}$, Fulvio Adorni $^{2}$, Simona G. Di Santo ${ }^{4}$, \\ Massimo Musicco $^{2,4}$, Nikolaos Scarmeas ${ }^{5,6}$ and Maria L. Correa Leite ${ }^{2}$ \\ ${ }^{1}$ Department of Environmental, Food and Nutritional Sciences, University of Milan, Via Celoria 2, 20101 Milan, Italy \\ ${ }^{2}$ Epidemiology and Biostatistics Unit, Institute of Biomedical Technology - National Research Council, Via Fratelli Cervi 93, \\ Segrate Milan, Italy \\ ${ }^{3}$ Department of Nutrition and Dietetics, Harokopio University, 70 Eleftheriou Venizelou Avenue, 17671 Athens, Greece \\ ${ }^{4}$ Department of Neuroscience, Santa Lucia Foundation IRCCS, Via Ardeatina 306, 00179 Rome, Italy \\ ${ }^{5}$ Department of Neurology, Taub Institute for Research in Alzheimer's Disease and the Aging Brain, Gertrude H. Sergievsky \\ Center, Columbia University, 622 West 168th Street, P and S\#16, NY 10032, USA \\ ${ }^{6}$ Department of Social Medicine, Psychiatry and Neurology, National and Kapodistrian, University of Athens, Eginition \\ Hospital 72 Vasilisis Sofias Avenue, 11528 Athens, Greece
}

(Submitted 25 July 2014 - Final revision received 13 January 2015 - Accepted 16 January 2015 - First published online 9 March 2015)

\begin{abstract}
The aim of the present analysis was to evaluate the association of the Mediterranean diet (MeDi), smoking habits and physical activity with all-cause mortality in an Italian population during a 20-year follow-up study. A total of 1693 subjects aged $40-74$ who enrolled in the study in 1991-5 were asked about dietary and other lifestyle information at baseline. Adherence to the MeDi was evaluated by the Mediterranean dietary score (MedDietScore). A healthy lifestyle score was computed by assigning 1 point each for a medium or high adherence to the MedDietScore, non-smoking and physical activity. Cox models were used to assess the associations between lifestyle factors and healthy lifestyle scores and all-cause mortality, adjusting for potential confounders. The final sample included 974 subjects with complete data and without chronic disease at baseline. During a median of 17.4 years of follow-up, 193 people died. Subjects with high adherence to the MedDietScore (hazard ratio (HR) $0.62,95 \%$ CI $0.43,0.89$ )), non-smokers (HR $0.71,95 \%$ CI $0.51,0.98$ ) and physically active subjects (HR $0.55,95 \%$ CI $0.36,0.82$ ) were at low risk of death. Each point increase in the MedDietScore was associated with a significant $5 \%$ reduction of death risk. Subjects with 1, 2 or 3 healthy lifestyle behaviours had a significantly 39, 56, and $73 \%$ reduced risk of death, respectively. A high adherence to MeDi, non-smoking and physical activity were strongly associated with a reduced risk of all-cause mortality in healthy subjects after long-term follow-up. This reduction was even stronger when the healthy lifestyle behaviours were combined.
\end{abstract}

Key words: Mediterranean diet: Lifestyle factors: Follow-up studies: All-cause mortality

The concept of the Mediterranean diet (MeDi) was originally conceived by Ancel Keys in the Seven Countries Study; the main finding of the prospective evaluation of that study's participants was that all-cause and CHD mortality rates were lower in cohorts where olive oil was the main dietary fat as compared to northern European cohorts ${ }^{(1)}$. The dietary pattern identified in these cohorts was characterised by an abundance of plant foods, including: fruits, mainly as a typical after-dinner dessert, vegetables, as either a main or a side dish, a lot of bread, other forms of cereal, legumes, nuts and seeds. Olive oil was the principal source of fat. The so-called MeDi also included moderate amounts of dairy products (principally cheese and yogurt), low to moderate amounts of fish and poultry, red meat in low amounts and wine, which was consumed moderately during meals ${ }^{(2)}$.

Since the Seven Countries Study, the beneficial effects of the MeDi on several chronic diseases, including CVD, cognitive decline and Alzheimer's Disease, cancer and diabetes, have been widely investigated and replicated in many observational studies and in some clinical trials ${ }^{(3-10)}$. Moreover, there is consistent evidence that MeDi reduces total mortality in both Mediterranean and non-Mediterranean populations ${ }^{(11-21)}$. In these prospective evaluations, the follow-up period varied from 5 to 14 years and adherence to MeDi was mostly assessed by scores that were population-specific; thus, the same score could reflect different patterns of food consumption in

Abbreviations: HR, hazard ratios; ICD, International Classification of Diseases; MedDietScore, Mediterranean dietary score; MeDi, Mediterranean diet. 
different studies, and no direct comparisons could be made between studies ${ }^{(22)}$.

Because adherence to a healthy diet such as the MeDi may also reflect adherence to a broader healthy lifestyle pattern, several cohort studies have investigated the effects of other lifestyle factors on total mortality ${ }^{(15,23-27)}$. However, to the best of our knowledge, only two studies have examined the combined effects of MeDi adherence, smoking status and physical activity on all-cause mortality ${ }^{(25,27)}$. There are no data thus far on the relationship between adherence to the MeDi, which is defined with an a priori index based on the principles of the Mediterranean pattern, and death with a relatively large follow-up period. Furthermore, little is known about the association between combined lifestyle behaviours, such as adherence to the MeDi, smoking habits and physical activity, and death in an apparently healthy population. Thus, the aims of the present analysis were (1) to investigate the relationship between adherence to an a priori MeDi score and its components and all-cause mortality in a 20-year follow-up study of an Italian cohort of healthy subjects; and (2) to evaluate the role that other lifestyle factors, such as smoking habits and physical activity, as well as a combined lifestyle index, play in death risk.

\section{Subjects and methods}

\section{Baseline}

Study population. Participants were recruited from 1991 to 1995. The study population was a simple random sample of subjects aged 40-74 years who were drawn from the residents' registries of two towns in Northern Italy (the Lombardy region). Participants were recruited to investigate the prevalence of and risk factors for major eye diseases ${ }^{(28)}$. An invitation letter was sent to 2882 subjects explaining the study objectives and procedures. Subsequently, each person was contacted by phone to collect consent and to book an appointment at the local hospital. Of the total individuals invited, 1693 (58.7\%) agreed to participate. During their hospital visit, participants were asked to fill out a series of questionnaires regarding medical and family history, past and current drug use and lifestyle habits. They also underwent a general clinical assessment that included anthropometric measurements (height, weight, body circumference and skinfold thickness) and arterial blood pressure measurement on both arms, and they provided a fasting blood sample for biochemical assessment (haematology, glycaemia and lipid profile).

The present study was conducted according to the guidelines laid down in the Declaration of Helsinki, and all procedures involving human subjects were approved by the ethical review board of the National Research Council of Segrate (Milan). Verbal informed consent was obtained from all subjects. Verbal consent was witnessed and formally recorded.

Assessment of dietary intake. Usual dietary intake during the year preceding recruitment was assessed with the use of an FFQ administered by a trained interviewer. This quantitative FFQ (a modification of Willett's questionnaire in the
Nurses' Health Study ${ }^{(29)}$ ) consisted of a list of 158 foods (items) arranged in thirteen categories (bread/cereal products, eggs/meats and processed meats, fish, dairy products, cheese, vegetables, oils/condiments/sauces, fruits, sweets and candies, beverages/coffee, alcohol, sugar and miscellaneous). Participants were asked to indicate how often they consumed a given item according to a seven-category frequency scale: never, 1-3 times/month; 1-2 times/week; 3-4 times/week; 1 time/d; $2-3$ times/d; or $4-5$ times/d. The amount of food consumed was assessed by the selection of a picture of a food portion. Daily intakes of nutrients were calculated using the food composition database, which was compiled for epidemiological studies in Italy ${ }^{(30)}$. Dietary information was missing for 251 participants.

Other assessments. Procedures for the anthropometric measurements have been described in detail elsewhere ${ }^{(31)}$. BMI was calculated as weight $(\mathrm{kg})$ divided by height squared $\left(\mathrm{m}^{2}\right)$. Education was classified with two levels: (1) primary school or less or (2) medium or high school. In relation to smoking status, subjects were categorised as either never or ever (former or current smokers).

A section of the questionnaire assessed occupational and leisure-time activities based on the time subjects spent watching TV (three categories: $<2 \mathrm{~h} / \mathrm{d}, 2-4 \mathrm{~h} / \mathrm{d}$ or more than $4 \mathrm{~h} / \mathrm{d}$ ) and the frequency with which they practiced sports (two categories: physically active (if they were engaged in at least one sport) or physically inactive)

For alcohol consumption, subjects were categorised as either (1) abstainers or light drinkers $(<10 \mathrm{~g} / \mathrm{d}$ for males and $<5 \mathrm{~g} / \mathrm{d}$ for females); (2) moderate drinkers $(10-50 \mathrm{~g} / \mathrm{d}$ for males and $5-25 \mathrm{~g} / \mathrm{d}$ for females); or (3) heavy drinkers ( $>50 \mathrm{~g} / \mathrm{d}$ for males and $>25 \mathrm{~g} / \mathrm{d}$ for females).

Physicians ascertained the presence of chronic diseases (CVD, diabetes and cancer) at baseline during the hospital visit. Participants who self-reported to have a disease or to use drugs for a specific disease were considered prevalent cases (for fifty-eight subjects, this information was missing). At baseline, $16.3 \%$ of subjects reported a diagnosis of CVD, $3.8 \%$ reported cancer and $6.6 \%$ reported diabetes. Because CVD, diabetes and cancer increase the risk of mortality and in order to minimise the possibility that diet or lifestyle were changed in response to morbidity, we excluded subjects who had a chronic disease at baseline.

Mediterranean dietary score. Adherence to the MeDi was evaluated with the MedDietScore ${ }^{(32)}$. Assessment was based on the weekly consumption of eleven foods and beverages groups: non-refined cereals (wholegrain bread and pasta, brown rice, etc.), fruits, vegetables, legumes, potatoes, fish, meat and meat products, poultry, full-fat dairy products (cheese, yogurt and milk), olive oil and alcohol. For the consumption of items that were presumed to closely follow the MeDi pattern (i.e. cereals, fruits, vegetables, legumes, potatoes, fish and olive oil), subjects who reported no consumption was assigned a score of 0 , and scores of 1-5 were assigned for rare to daily consumption. For the consumption of foods that were presumed to diverge from this diet pattern (i.e. meat and meat products, poultry and full-fat dairy products), subjects were assigned scores on a reverse scale 
(i.e. from 5 when they reported no consumption to 0 when they reported almost daily consumption). For alcohol intake, a score of 5 was assigned for consumption of less than $300 \mathrm{ml}$ of alcohol/d, a score of 0 was assigned for no consumption or for consumption of $>700 \mathrm{ml} / \mathrm{d}$ and scores of $4-1$ were assigned for consumption of 600-700, 500-600, 400-500 and $300-400 \mathrm{ml} / \mathrm{d}$ ( $100 \mathrm{ml}$ have $12 \mathrm{~g}$ of ethanol concentration), respectively. The total possible score ranged from 19 to 45 . Higher values of this diet score indicated greater adherence to the MeDi. In the present study, information about nonrefined cereals was not collected, so we replaced non-refined grains with all kinds of pasta, rice and flour.

Healthy lifestyle score. In order to evaluate the combined effect of lifestyle factors, a lifestyle score was calculated by adding the individual values for the MedDietScore, physical activity level and smoking status. The MedDietScore was categorised in tertiles. Individuals scored 0 points if they belonged to the lowest tertile, and they scored 1 point if they belonged to the medium or highest tertile. For smoking, individuals were considered to be at low risk (1 point) if they had never smoked and at high risk ( 0 points) if they were former or current smokers. Individuals who were physically inactive were considered to be at high risk ( 0 points), and subjects who were physically active were considered at low risk (1 point). The total possible score ranged from 0 to 3 .

\section{Follow-up}

Ascertainment of vital status and outcome. To retrieve subjects with available data on vital status, a deterministic record linkage between the baseline cohort and the Regional Registries of the Informative System of the Local Health Authority of Milan 1 was developed using two match keys: first, by matching fiscal code or health card number (step 1) and second, by matching full name and date of birth (step 2). A check was then performed for duplicate cases or discrepancies. Of the initial 1693 study participants, 1604 were retrieved after the record-linkage procedure; of those, thirty-five migrated before the follow-up date (31 October 2012).

The final cohort with complete data on vital status was followed up for death using the regional Registry of Mortality. Causes of death were coded and classified according to the International Classification of Diseases, ninth and tenth revisions (ICD-9 and ICD-10), coding system. ICD-9 codes 390459.9 and ICD-10 codes I00-I99 were used for defining specific-cause death for CVD, and ICD-9 codes 140-239.9 and ICD-10 codes C00-D99 were used for defining cancer. Mortality from other causes was defined as total deaths minus deaths from unknown causes, CVD and cancer. The final sample that was analysed consisted of 974 subjects ( 483 men and 491 women) who did not previously have chronic diseases (CVD, cancer or diabetes), with vital status ascertained and with complete dietary data (Fig. 1).

\section{Statistical analysis}

Continuous variables were presented as means and standard deviations. Frequency and percentages were reported for categorical variables. Follow-up included the time from enrolment to time of death, migration out of the region or the end

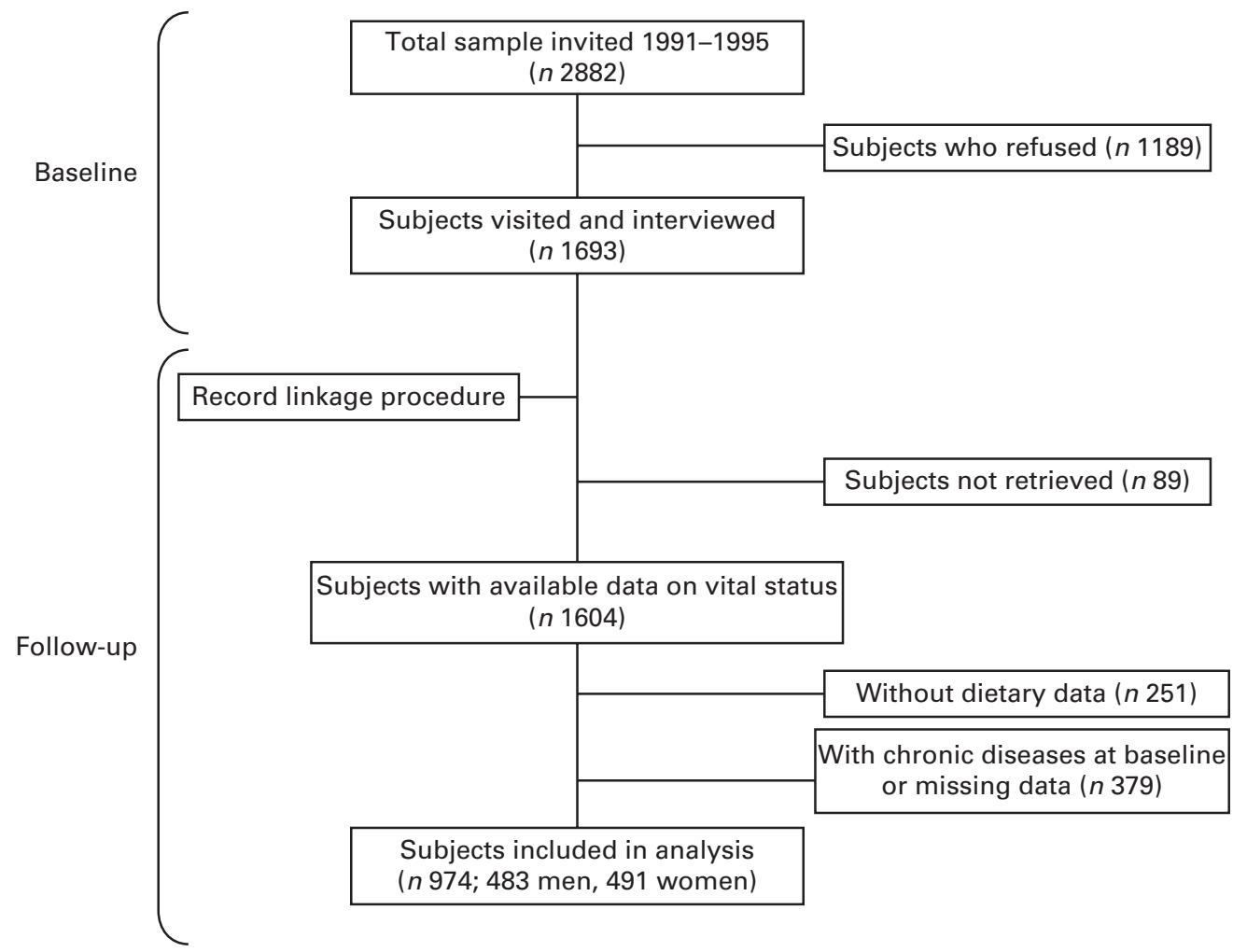

Fig. 1. Flow chart describing the steps involved in establishing the final study population. 
of follow-up, whichever came first. Cox proportional hazard regression models were used to estimate the effects of diet, smoking status and physical activity as well as their combined effect on all-cause mortality. Relative risks were estimated as hazard ratios (HR) with $95 \% \mathrm{CI}$.

Initial crude analyses were performed. Further models were adjusted for age at baseline (continuous) and sex (male as reference). Fully adjusted models included education level (primary school or less as reference), smoking status (never smokers as reference), physical activity (no as reference), BMI (continuous, $\mathrm{kg} / \mathrm{m}^{2}$ ), time spent watching $\mathrm{TV}^{(33)}(<2 \mathrm{~h} / \mathrm{d}$ as reference) and total daily energy intake (continuous, $\mathrm{kJ} / \mathrm{d}$ ).

The HR for MedDietScore were estimated using the subjects in the lowest tertiles of adherence as the reference group. In addition to examining the relationship between the MedDietScore and all-cause mortality, we also investigated the effects of each single component of the diet score on death. The median of each component was used as a cut-off point, and the value below the 50th percentile was considered the reference category. First, we evaluated the effects of each of the eleven food components, including age, sex, education level, BMI, physical activity, smoking status, time spent watching TV and energy intake, in the model. Then, all of the components were introduced simultaneously in the model. The association between healthy lifestyle score and death was evaluated in multivariate-adjusted Cox regression models that controlled for age, sex, education level, BMI, time spent watching TV and energy intake. Subjects who scored 0 points on the lifestyle score were considered the reference group. All statistical analyses were performed using SPSS software package version 19.0 (IBM Corporation, 2010; IBM SPSS Statistics for Macintosh).

\section{Results}

Baseline characteristics of the cohort and all-cause mortality data according to sex are presented in Table 1 . The mean age of males and females was 55.8 and 55.7 years, respectively. The BMI values were almost similar between the two groups. Males were more educated and were more frequently former or current smokers and heavy drinkers as compared to females. Overall, $21.5 \%$ of the subjects were physically active. (Gymnastics, swimming, using an exercise bike or cycling, tennis and running were the most commonly practiced sports). The median follow-up with the participants was $17 \cdot 4$ years, with a minimum and maximum follow-up of 4 months and 21.4 years, respectively. During the follow-up period, 16926 person-years were accrued, and 193 deaths occurred (131 men and sixty-two women). The main cause of death for both males and females was cancer $(34.4$ and $30.6 \%$, respectively), followed by CVD (22.9 and $22.6 \%$, respectively); twenty-one subjects died from other causes, and sixty-four died from unknown causes.

We compared the subjects who were included in the analysis ( $n$ 974) with those who were excluded because they previously had chronic diseases ( $n$ 379). The excluded subjects were significantly older, had higher BMI, were less
Table 1. Baseline characteristics and 20-year death data of the Italian cohort by sex ( $n 974,483$ males and 491 females)

(Mean values and standard deviations)

\begin{tabular}{|c|c|c|c|c|}
\hline & \multicolumn{2}{|c|}{$\begin{array}{c}\text { Males } \\
(49.6 \%)\end{array}$} & \multicolumn{2}{|c|}{$\begin{array}{l}\text { Females } \\
(50.4 \%)\end{array}$} \\
\hline & $n$ & $\%$ & $n$ & $\%$ \\
\hline \multicolumn{5}{|l|}{ Age at baseline } \\
\hline Mean & $55 \cdot 8$ & & $55 \cdot 7$ & \\
\hline SD & $7 \cdot 9$ & & 7.5 & \\
\hline \multicolumn{5}{|l|}{$\mathrm{BMI}\left(\mathrm{kg} / \mathrm{m}^{2}\right)$} \\
\hline Mean & $26 \cdot 8$ & & $26 \cdot 8$ & \\
\hline SD & 3.5 & & 4.6 & \\
\hline \multicolumn{5}{|l|}{ Education level } \\
\hline Primary school or less & 212 & 43.9 & 309 & $62 \cdot 9$ \\
\hline Medium school or graduate & 271 & $56 \cdot 1$ & 182 & $37 \cdot 1$ \\
\hline \multicolumn{5}{|l|}{ Smoking status } \\
\hline Never & 145 & $30 \cdot 0$ & 350 & $71 \cdot 3$ \\
\hline Ever & 338 & $70 \cdot 0$ & 141 & $28 \cdot 7$ \\
\hline \multicolumn{5}{|l|}{ Alcohol intake $(g / d)^{*}$} \\
\hline Abstainers or light drinkers & 107 & $22 \cdot 2$ & 287 & 58.5 \\
\hline Moderate drinkers & 197 & $40 \cdot 8$ & 117 & $23 \cdot 8$ \\
\hline Heavy drinkers & 179 & $37 \cdot 1$ & 87 & $17 \cdot 7$ \\
\hline \multicolumn{5}{|l|}{ Physical activity } \\
\hline Inactive & 371 & $76 \cdot 8$ & 394 & $80 \cdot 2$ \\
\hline Active & 112 & $23 \cdot 2$ & 97 & $19 \cdot 8$ \\
\hline \multicolumn{5}{|l|}{ MedDietScore } \\
\hline Low & 142 & $29 \cdot 4$ & 236 & $48 \cdot 1$ \\
\hline Medium & 164 & 34.0 & 142 & 28.9 \\
\hline High & 177 & $36 \cdot 6$ & 113 & $23 \cdot 0$ \\
\hline All deaths & 131 & $27 \cdot 1$ & 62 & $12 \cdot 6$ \\
\hline \multicolumn{5}{|l|}{ Causes of death } \\
\hline Cancer & 45 & 34.4 & 19 & $30 \cdot 6$ \\
\hline CVD & 30 & $22 \cdot 9$ & 14 & $22 \cdot 6$ \\
\hline Other & 16 & $12 \cdot 2$ & 5 & $8 \cdot 1$ \\
\hline Unknown & 40 & $30 \cdot 5$ & 24 & $38 \cdot 7$ \\
\hline
\end{tabular}

MedDietScore, Mediterranean dietary score.

${ }^{*}$ Abstainers or light drinkers $(<10 \mathrm{~g} / \mathrm{d}$ for males and $<5 \mathrm{~g} / \mathrm{d}$ for females); moderate drinkers $(10-50 \mathrm{~g} / \mathrm{d}$ for males and $5-25 \mathrm{~g} / \mathrm{d}$ for females); heavy drinkers ( $>50 \mathrm{~g} / \mathrm{d}$ for males and $>25 \mathrm{~g} / \mathrm{d}$ for females).

educated, were more physically inactive and had higher mortality in comparison with the subjects who were included in the analyses (data not shown)

Table 2 presents the proportional HR with 95\% CI for allcause mortality in relation to physical activity, smoking status and tertiles of MedDietScore. In the age- and sexadjusted model, engaging in physical activity (HR 0.56, 95\% CI $0.38,0.84$ ) and never having smoked (HR 0.71, 95\% CI $0.51,0.97)$ were factors that were significantly inversely associated with all-cause mortality. In the multivariate model that was adjusted for age, sex, education, BMI and energy intake, physically active subjects and never smokers had a significant $45 \%$ (HR 0.55, 95\% CI 0.36, 0.82) and 29\% (HR 0.71, 95\% CI $0.51,0.98)$ lower risk of death. High adherence to the MeDi significantly reduced the risk of death in the age- and sex-adjusted model (HR 0.64, 95\% CI $0.45,0.91)$ and in the multivariate model (HR 0.62, 95\% CI 0.43, 0.89). When we included MedDietScore in the model as a continuous variable, the HR was 0.95 (95\% CI 0.92, 0.98), which meant there was a $5 \%$ reduction of all-cause mortality risk for each 1-point increase in the MedDietScore. No interactions were found between the MedDietScore and the covariates included in the model. 
Table 2. The association between smoking status, physical activity and adherence (increasing tertiles) to MedDietScore (Mediterranean dietary score) and 20-year all-cause mortality in the Italian cohort

(Hazard ratios (HR) and $95 \%$ confidence intervals)

\begin{tabular}{|c|c|c|c|c|c|c|c|}
\hline & $\begin{array}{c}\text { No. of } \\
\text { subjects }\end{array}$ & Cases & Person-years & $\begin{array}{c}\text { Age- and } \\
\text { sex-adjusted HR }\end{array}$ & $95 \% \mathrm{Cl}$ & $\begin{array}{c}\text { Multivariate } \\
\qquad \mathrm{HR}^{\star}\end{array}$ & $95 \% \mathrm{Cl}$ \\
\hline \multicolumn{8}{|c|}{ Physical activity } \\
\hline Inactive & 765 & 165 & 13156 & 1 & Reference & 1 & Reference \\
\hline Active & 209 & 28 & 3770 & 0.56 & $0.38,0.84$ & 0.55 & $0.36,0.82$ \\
\hline \multicolumn{8}{|c|}{ Smoking status } \\
\hline Ever & 479 & 109 & 8125 & 1 & Reference & 1 & Reference \\
\hline Never & 495 & 84 & 8801 & 0.71 & $0.51,0.97$ & 0.71 & $0.51,0.98$ \\
\hline \multicolumn{8}{|c|}{ MedDietScore } \\
\hline Low & 378 & 80 & 6597 & 1 & Reference & 1 & Reference \\
\hline Medium & 306 & 61 & 5220 & 0.80 & $0.57,1.12$ & 0.79 & $0.56,1.12$ \\
\hline \multirow[t]{2}{*}{ High } & 290 & 52 & 5109 & 0.64 & $0.45,0.91$ & 0.62 & $0.43,0.89$ \\
\hline & & & & \multicolumn{2}{|c|}{$P$ for trend $=0.014$} & \multicolumn{2}{|c|}{$P$ for trend $=0.010$} \\
\hline
\end{tabular}

* Model additionally adjusted for education level, BMI, time spent watching TV (h/d) and energy intake (kJ/d).

Figure 2 shows the adjusted estimated risks of all-cause mortality for single lifestyle factors (physical activity, smoking status and adherence to MeDi). Dots and vertical lines indicate HR and $95 \%$ CI. The $P$ value for the trend test refers to tertiles of MeDi adherence.

Table 3 reports the associations between the eleven components of the MedDietScore and all-cause mortality, contrasting high and low consumption and controlling for potential confounders. High consumption of fruits was inversely associated with all-cause mortality (HR 0.71, 95\% CI 0.53, 0.96); the estimates did not change when all of the components were included simultaneously in the model (HR 0.70, 95\% CI $0.51,0.95)$. Death risk was not singularly associated with the other components.

Keeping the subjects with a zero healthy lifestyle score as the reference group, the fully adjusted HR estimated in the 1 -score, the 2-score and the 3-score groups were 0.61 (95\% CI $0.41,0.89), 0.44(95 \%$ CI $0.29,0.67)$ and 0.27 (95\% CI $0 \cdot 12,0.61)$, respectively. The test for a linear trend was statistically significant with $P<0 \cdot 001$. Cumulative survival curves adjusted for healthy lifestyle scores are presented in Fig. 3.

Sensitivity analyses were performed to determine the robustness of the present results. The statistical analyses were repeated replacing the MedDietScore with the Mediterranean Diet Score used by Trichopoulou et al. ${ }^{(12)}$ in the models, and the corresponding healthy lifestyle score was recalculated. We found that the results did not change significantly. We observed that subjects in the highest tertile of Mediterranean Diet Score adherence had a low risk of death (HR 0.69, $95 \%$ CI $0 \cdot 46,1 \cdot 03, P$ for trend of $0 \cdot 07$ ). Subjects with 1,2 or 3 healthy lifestyle behaviours had a significant reduced risk of death of 36,52 and $71 \%$, respectively.

Furthermore, in order to minimise the potential bias of undiagnosed chronic diseases at the time of enrolment, a sensitivity analysis was carried out excluding subjects who died in the first 2 years of follow-up ( $n 8$ ), and the estimated relative risks did not change (data not shown).

\section{Discussion}

The present analysis evaluated the association between the adherence to MeDi and other lifestyle factors and the risk of all-cause mortality in a healthy Italian population during a 20-year follow-up study. We found that subjects with a high adherence to MeDi, non-smokers and physically active subjects were at a lower risk of death. A healthy lifestyle score

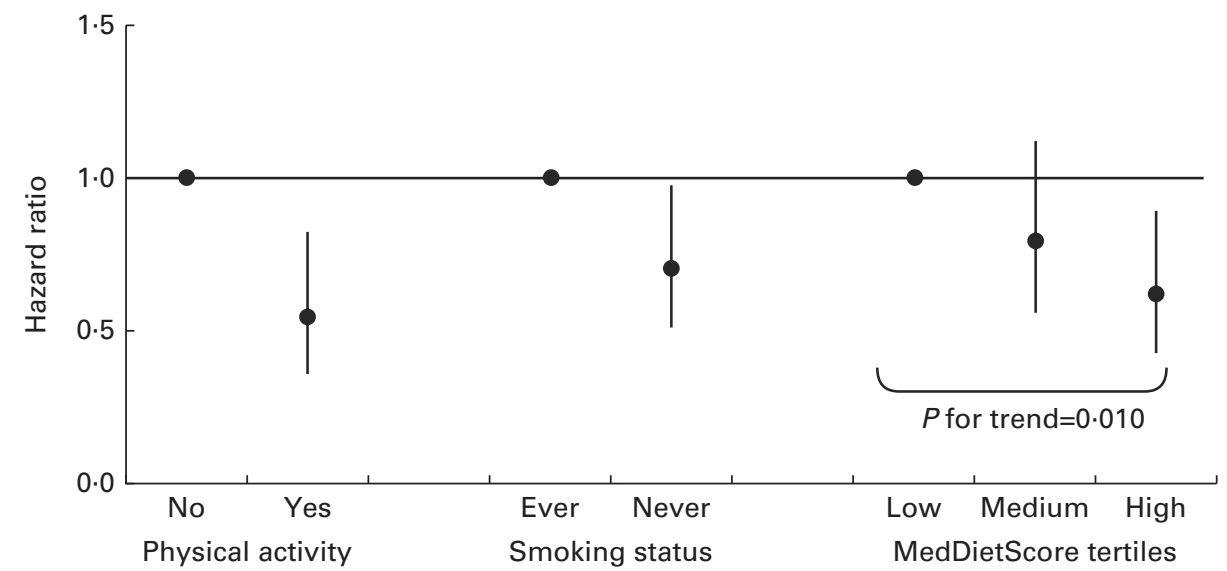

Fig. 2. Adjusted hazard ratios (HR) $(95 \% \mathrm{Cl})$ of all-cause mortality for single lifestyle factors. Adjusted for age, sex, education level, BMI, time spent watching TV $(\mathrm{h} / \mathrm{d})$ and energy intake $(\mathrm{kJ} / \mathrm{d})$. MedDietScore, Mediterranean dietary score. 
Table 3. All-cause mortality-adjusted hazard ratios (HR) associated with intake of the different components of the MedDietScore (Mediterranean dietary score)

(Hazard ratios and $95 \%$ confidence intervals)

\begin{tabular}{|c|c|c|c|c|}
\hline \multirow{2}{*}{$\begin{array}{l}\text { MedDietScore } \\
\text { (serve/week) components }\end{array}$} & \multicolumn{4}{|c|}{ All-cause mortality-adjusted HR } \\
\hline & $\mathrm{HR}^{*}$ & $95 \% \mathrm{Cl}$ & $\mathrm{HR} \dagger$ & $95 \% \mathrm{Cl}$ \\
\hline \multicolumn{5}{|l|}{ Vegetables } \\
\hline$\leq$ Median & 1 & Reference & 1 & Reference \\
\hline > Median & 0.92 & $0.68,1.24$ & 0.93 & $0.68,1.27$ \\
\hline \multicolumn{5}{|l|}{ Legumes } \\
\hline$\leq$ Median & 1 & Reference & 1 & Reference \\
\hline$>$ Median & 1.07 & $0.80,1.44$ & 1.08 & $0.79,1.48$ \\
\hline \multicolumn{5}{|l|}{ Fruits } \\
\hline$\leq$ Median & 1 & Reference & 1 & Reference \\
\hline > Median & 0.71 & $0.52,0.96$ & 0.70 & $0.51,0.95$ \\
\hline \multicolumn{5}{|l|}{ Cereals } \\
\hline$\leq$ Median & 1 & Reference & 1 & Reference \\
\hline > Median & 0.93 & $0 \cdot 68,1 \cdot 27$ & 0.91 & $0.66,1.26$ \\
\hline \multicolumn{5}{|l|}{ Potatoes } \\
\hline$\leq$ Median & 1 & Reference & 1 & Reference \\
\hline$>$ Median & 0.99 & $0.72,1.34$ & 0.99 & $0.73,1.36$ \\
\hline \multicolumn{5}{|l|}{ Fish and seafood } \\
\hline$\leq$ Median & 1 & Reference & 1 & Reference \\
\hline$>$ Median & 0.99 & $0.74,1.34$ & 0.98 & $0.72,1.35$ \\
\hline \multicolumn{5}{|l|}{ Dairy products } \\
\hline$\leq$ Median & 1 & Reference & 1 & Reference \\
\hline$>$ Median & 1.26 & $0.93,1.72$ & 1.27 & $0.93,1.73$ \\
\hline \multicolumn{5}{|l|}{ Red meat and meat products } \\
\hline$\leq$ Median & 1 & Reference & 1 & Reference \\
\hline$>$ Median & 1.21 & $0.89,1.63$ & 1.23 & $0.91,1.68$ \\
\hline \multicolumn{5}{|l|}{ Poultry } \\
\hline$\leq$ Median & 1 & Reference & 1 & Reference \\
\hline > Median & $1 \cdot 19$ & $0.89,1.59$ & $1 \cdot 22$ & $0.90,1.65$ \\
\hline \multicolumn{5}{|l|}{ Olive oil } \\
\hline$\leq$ Median & 1 & Reference & 1 & Reference \\
\hline$>$ Median & 0.98 & $0.73,1.31$ & 0.99 & $0.74,1.34$ \\
\hline \multicolumn{5}{|l|}{ Ethanol intake } \\
\hline$\leq$ Median & 1 & Reference & 1 & Reference \\
\hline$>$ Median & 1.01 & $0.72,1.42$ & 1.01 & $0.71,1.42$ \\
\hline
\end{tabular}

that combined all of these factors showed a strong inverse relation with all-cause mortality.

Subjects in the highest tertile of MedDietScore had a $38 \%$ reduction of death risk as compared to subjects in the lowest tertile, and there was a $5 \%$ reduction of death risk for each 1-point increase in the MedDietScore. The present results are consistent with other European and US prospective cohort studies that reported inverse associations between an adherence to the MeDi and total mortality ${ }^{(11-21)}$. Two recent meta-analyses summarised the prospective cohort studies that evaluated the association between the $\mathrm{MeDi}^{(11,12)}$ and overall mortality and reported an $8 \%$ reduction for a 2 -point increase in adherence to the $\mathrm{MeDi}^{(34,35)}$. Furthermore, the present results have proven to be not sensitive to the substitution of the MedDietScore with another Mediterranean diet-related score, such as the Mediterranean Diet Score. The biological explanations for the protective role of the MeDi, which is rich in plant-based foods, might include both its antioxidant and anti-inflammatory effects ${ }^{(36,37)}$.
When we evaluated the role of the single components of the MedDietScore, we found that subjects with a high intake of fruits had a $30 \%$ reduction of risk for all-cause mortality; however, no strong associations were evident for the other score components. We speculate that the high interaction between the individual components of the score and the fact that single foods may have small effects that appear only when all of the components are included is a pattern ${ }^{(38)}$. Still, by analysing the effects of specific components, one might miss associations between diet and disease, because the effects of the individual components are examined against a background of average risk that is associated with other nutrients or foods ${ }^{(39)}$.

Participants who were engaged in at least one sport had a reduction in death risk of $45 \%$. A recent systematic review and meta-analysis on cohort studies reported that increasing levels of total and domain-specific physical activity reduced the risk of all-cause mortality by $35 \%$, with stronger associations for exercise, sports, leisure-time activities and activities of daily living, rather than occupational and transport-related activity $^{(40)}$. In another meta-analysis on longitudinal studies, the authors found that $2.5 \mathrm{~h} /$ week (equivalent to $30 \mathrm{~min}$ daily of moderate-intensity activity $5 \mathrm{~d}$ /week) was associated with a reduction in mortality risk of $19 \%$ as compared to no activity, and $7 \mathrm{~h} /$ week of moderate activity reduced the mortality risk by $24 \%$ as compared to no activity ${ }^{(41)}$. The mechanisms underlying this protective effect could involve the improvement of the cardio-metabolic profile, which results in a reduced risk for vascular diseases that are associated with high mortality risk ${ }^{(42,43)}$. In addition, regular physical activity decreases the susceptibility of cells and tissues to

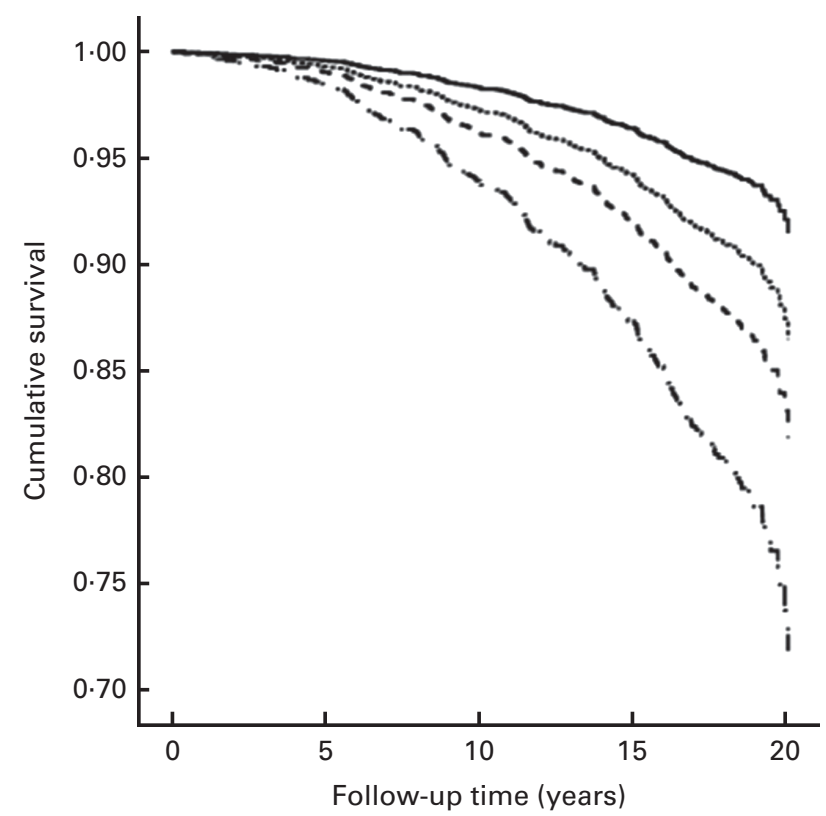

Fig. 3. Adjusted cumulative survival curve of all-cause mortality by healthy lifestyle score (range: $0-3$ ), including the following factors: adherence to the Mediterranean diet, physical activity and smoking habits. Adjusted for age, sex, education level, BMI, time spent watching TV $(\mathrm{h} / \mathrm{d})$ and energy intake

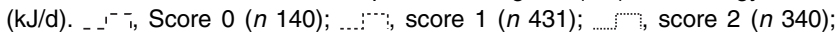
$\neg$, score 3 ( $n$ 63). 
oxidative stress, increases vascularisation and enhances energy metabolism ${ }^{(44)}$

Never smokers had a $29 \%$ lower risk of death than ever smokers. Other cohort studies reported similar risk estimates $^{(45)}$. Tobacco smoke is a mixture of several toxic compounds that are involved in disease development by different mechanisms ${ }^{(46)}$. Smoking remains the leading preventable cause of disease and death, and approximately 6 million deaths worldwide every year are attributed to smoking ${ }^{(47)}$.

When considering the combined effects of diet, physical activity and smoking status, a higher number of healthy lifestyle factors was associated with lower all-cause mortality. Compared to subjects in the lowest healthy lifestyle score group, subjects scoring a 1, 2 or 3 points had a 39, 56, and $73 \%$ reduction in their risk of death during follow-up, respectively. The authors of a recent meta-analysis ${ }^{(26)}$ reported a reduction in mortality by $66 \%(95 \%$ CI 58,73$)$ for a combination of different healthy lifestyle behaviours, including smoking, BMI, physical activity, alcohol habits and diet, but only a few previous studies have considered a MeDi food pattern. In the HALE Project Study, which was carried out on individuals aged 70-90 years, Knoops et al. ${ }^{(15)}$ found that the combination of a high adherence to MeDi, moderate alcohol intake, high levels of physical activity and non-smoking lowered the all-cause mortality rate to 0.35 (95\% CI $0 \cdot 28$, $0 \cdot 44$ ) during 10 years of follow-up. In a cohort of 120852 subjects aged 55-69, the combination of high MeDi adherence, normal BMI, non-smoking and physical activity was associated with a $31 \%$ lower risk of death in women, but not in men, over a period of 10 years $^{(48)}$. In another large cohort of individuals aged 50-71 who did not have a chronic disease at baseline, adherence to four low-risk lifestyle factors, including abdominal leanness, performing the recommended amount of physical activity, long-term non-smoking and a high adherence to the MeDi, was associated with a strong reduction in the risk of mortality (HR $0.27,95 \%$ CI $0 \cdot 25,0 \cdot 29$ ) during 12.5 years of follow-up as compared to subjects who did not adhere to any of these factors ${ }^{(24)}$. Ahmed et al. ${ }^{(23)}$ found that participants aged 44-84 ( $n$ 6229) who adopted four healthy behaviours (smoking avoidance, regular physical activity, adherence to a Mediterranean-style diet and normal weight maintenance) had an approximately 80\% lower death rate than participants who did not adopt any healthy behaviours during 10 years of follow-up. In the SENECA Study, 1281 subjects aged 70-75 were followed for 10 years, and three unhealthy behaviours (having a low adherence to MeDi, smoking, and being physically inactive) resulted in a three- to fourfold increased risk of mortality ${ }^{(27)}$. Finally, in the Italian Rural Area Seven Country Study, a combination of three unhealthy risk factors (smoking, a sedentary lifestyle and a dietary pattern far from the Mediterranean style) was associated with 4.8-year life loss in a 20-year follow-up and a 10-7-year life loss in a 40-year follow-up ${ }^{(25)}$. Although it was characterised by a long follow-up period, that study was carried out with a cohort of only middle-aged men, and eating habits were derived through an a posteriori approach. Compared to an a priori method, this approach depends on a cohort's characteristics: the pattern derived is highly specific to the diet of the population of interest, and it is greatly influenced by the authors' subjective choices during the analyses ${ }^{(49)}$; thus, it cannot be generalised to the general target population and is not applicable to other countries.

Strengths of the present study include its prospective design with a long follow-up as well as the small number of participants who were lost during follow-up, which limited the possibility of selection bias. We investigated a range of potential confounding factors, including age, sex, education level, BMI and energy intake, which allowed us to control for their potential confounding effects in the analysis. Adherence to the MeDi was evaluated by means of a score that is in agreement with the principles of the Mediterranean pattern and was not specific to the dietary consumption of the population studied. Advantages of the MedDietScore include the weighting of the selected food groups based on the frequency of consumption (thresholds were chosen according to an a priori hypothesis) and regardless of the consumptions of the sample studied ${ }^{(22)}$.

Finally, we excluded subjects with chronic diseases at enrolment, which thus reduced the potential effect of disease on lifestyle behaviours such as diet or physical activity. The exclusion of subjects with co-morbidities and those who died very early did not affect the associations we observed.

A limitation of the present study is that the single dietary intake assessment administered in adult life might not have reflected the long-term dietary intake of the participants. Physical activity was self-reported and also assessed only at one single time during adulthood. We considered subjects to be physically active when they were engaged in at least one sport, and we did not take into account free-living physical activity, including standing, walking, sitting or moving around; therefore, we might have underestimated the level of physical activity of the participants. The sample size in the present cohort was relatively moderate; however, this potential limitation was counterbalanced by the long followup period. We also cannot rule out the possibility of residual confounding as a result of other factors that were not considered in the present study, even though we adjusted for the main known risk factors.

In summary, the present findings support the hypothesis that a healthy lifestyle, i.e. adherence to a Mediterranean dietary pattern, abstinence from smoking and regular engagement in physical activity, has a positive effect on reducing the risk of all-cause mortality, especially when the combined effect of all three lifestyle behaviours was considered. These results seem to be clinically relevant in terms of public health, particularly for policy makers that aim to reduce the risk of all-cause mortality in the healthy general population. Primary prevention strategies that encourage healthy lifestyle behaviours will be necessary in order to improve individual and community health.

\section{Acknowledgements}

The present study was made possible by the contributions of the original investigators and the team who recruited the participants at baseline. 
The contributions of the authors are as follows: F. P. carried out the nutritional and statistical analyses, interpreted the results and wrote the original manuscript; M. L. C. L., M. Y. and C. A. A. helped to analyse the nutritional data and interpret the results; M. Y. and C. A. A. assisted with writing the manuscript; F. A. contributed to the statistical analyses; S. G. D. S. participated in interpreting the results; M. M., M. Y., N. S. and M. L. C. L. critically revised the manuscript for important intellectual content. All authors read and approved the final version of the manuscript.

None of the authors has any conflicts of interest to declare.

\section{References}

1. Keys A, Menotti A, Karvonen MJ, et al. (1986) The diet and 15-year death rate in the seven countries study. $A m \mathrm{~J}$ Epidemiol 124, 903-915.

2. Willet W (1995) Mediterranean diet pyramid: a cultural model for healthy eating. Am J Clin Nutr 61, 1402S-1406S.

3. Martinez-Gonzalez MA, Bes-Rastrollo M, Serra-Majem L, et al. (2009) Mediterranean food pattern and the primary prevention of chronic disease: recent developments. Nutr Rev 67, Suppl. 1, S111-S116.

4. Lourida I, Soni M, Thompson-Coon J, et al. (2013) Mediterranean diet, cognitive function, and dementia: a systematic review. Epidemiology 24, 479-489.

5. Benetou V, Trichopoulou A, Orfanos P, et al. (2008) Conformity to traditional Mediterranean diet and cancer incidence: the Greek EPIC cohort. Br J Cancer 99, 191-195.

6. Serra-Majem L, Roman B \& Estruch R (2006) Scientific evidence of interventions using the Mediterranean diet: a systematic review. Nutr Rev 64, S27-S47.

7. Vincent-Baudry S, Defoort C, Gerber M, et al. (2005) The Medi-RIVAGE study: reduction of cardiovascular disease risk factors after a 3-mo intervention with a Mediterraneantype diet or a low-fat diet. Am J Clin Nutr 82, 964-971.

8. Estruch R, Martinez-Gonzalez MA, Corella D, et al. (2006) Effects of a Mediterranean-style diet on cardiovascular risk factors: a randomized trial. Ann Intern Med 145, 1-11.

9. Salas-Salvado J, Bullo M, Babio N, et al. (2011) Reduction in the incidence of type 2 diabetes with the Mediterranean diet: results of the PREDIMED-Reus nutrition intervention randomized trial. Diabetes Care 34, 14-19.

10. Estruch R, Ros E, Salas-Salvado J, et al. (2013) Primary prevention of cardiovascular disease with a Mediterranean diet. N Engl J Med 368, 1279-1290.

11. Trichopoulou A, Kouris-Blazos A, Wahlqvist ML, et al. (1995) Diet and overall survival in elderly people. BMJ 311, 1457-1460.

12. Trichopoulou A, Costacou T, Bamia C, et al. (2003) Adherence to a Mediterranean diet and survival in a Greek population. $N$ Engl J Med 348, 2599-2608.

13. Mitrou PN, Kipnis V, Thiebaut AC, et al. (2007) Mediterranean dietary pattern and prediction of all-cause mortality in a US population: results from the NIH-AARP Diet and Health Study. Arch Intern Med 167, 2461-2468.

14. Lagiou P, Trichopoulos D, Sandin S, et al. (2006) Mediterranean dietary pattern and mortality among young women: a cohort study in Sweden. Br J Nutr 96, 384-392.

15. Knoops KT, de Groot LC, Kromhout D, et al. (2004) Mediterranean diet, lifestyle factors, and 10-year mortality in elderly European men and women: the HALE project. JAMA 292, $1433-1439$.
16. Trichopoulou A, Orfanos P, Norat T, et al. (2005) Modified Mediterranean diet and survival: EPIC-elderly prospective cohort study. BMJ 330, 991.

17. Trichopoulou A, Bamia C \& Trichopoulos D (2009) Anatomy of health effects of Mediterranean diet: Greek EPIC prospective cohort study. BMJ 338, b2337.

18. Martinez-Gonzalez MA, Guillen-Grima F, De Irala J, et al. (2012) The Mediterranean diet is associated with a reduction in premature mortality among middle-aged adults. $J$ Nutr 142, 1672-1678.

19. Buckland G, Agudo A, Travier N, et al. (2011) Adherence to the Mediterranean diet reduces mortality in the Spanish cohort of the European Prospective Investigation into Cancer and Nutrition (EPIC-Spain). Br J Nutr 106, 1581-1591.

20. Tognon G, Lissner L, Saebye D, et al. (2014) The Mediterranean diet in relation to mortality and CVD: a Danish cohort study. Br J Nutr 111, 151-159.

21. McNaughton SA, Bates CJ \& Mishra GD (2012) Diet quality is associated with all-cause mortality in adults aged 65 years and older. $J$ Nutr 142, 320-325.

22. Feart C, Samieri C, Alles B, et al. (2013) Potential benefits of adherence to the Mediterranean diet on cognitive health. Proc Nutr Soc 72, 140-152.

23. Ahmed HM, Blaha MJ, Nasir K, et al. (2013) Low-risk lifestyle, coronary calcium, cardiovascular events, and mortality: results from MESA. Am J Epidemiol 178, 12-21.

24. Behrens G, Fischer B, Kohler S, et al. (2013) Healthy lifestyle behaviors and decreased risk of mortality in a large prospective study of U.S. women and men. Eur J Epidemiol 28, 361-372.

25. Menotti A, Puddu PE, Lanti M, et al. (2014) Lifestyle habits and mortality from all and specific causes of death: 40-year follow-up in the Italian Rural Areas of the Seven Countries Study. J Nutr Health Aging 18, 314-321.

26. Loef M \& Walach H (2012) The combined effects of healthy lifestyle behaviors on all cause mortality: a systematic review and meta-analysis. Prev Med 55, 163-170.

27. Haveman-Nies A, de Groot L, Burema J, et al. (2002) Dietary quality and lifestyle factors in relation to 10-year mortality in older Europeans: the SENECA study. Am J Epidemiol 156, 962-968.

28. Nicolosi A (1997) Le indagini di popolazione sulla prevalenza delle principali malattie oculari in Italia (Population surveys on prevalence of major eye diseases in Italy). In L'epidemiologia oftalmica in Italia (Ophthalmic Epidemiology in Italy), pp. 43-70 [L Cerulli, M Miglior and F Ponte, editors]. Rome: Editore I.N.C. Innovation-NewsCommunication.

29. Willet WC (1998) Nurses' Health Study Dietary questionnaire: appendix 5-1. In Nutritional Epidemiology, 2nd ed, pp. 95-97. New York: Oxford University Press.

30. Salvini S, Parpinel M, Gnagnarella P, et al. (1998) Banca dati di composizione degli alimenti per studi epidemiologici in Italia (Database of Food Composition for Epidemiological Studies in Italy). Milan: Istituto Europeo di Oncologia.

31. Leite ML \& Nicolosi A (2006) Lifestyle correlates of anthropometric estimates of body adiposity in an Italian middle-aged and elderly population: a covariance analysis. Int $J$ Obes (Lond) 30, 926-934.

32. Panagiotakos DB, Pitsavos C, Arvaniti F, et al. (2007) Adherence to the Mediterranean food pattern predicts the prevalence of hypertension, hypercholesterolemia, diabetes and obesity, among healthy adults; the accuracy of the MedDietScore. Prev Med 44, 335-340.

33. Basterra-Gortari F (2014) Television viewing, computer use, time driving and all-cause mortality: the SUN cohort. $J \mathrm{Am}$ Heart Assoc 3, e000864. 
34. Sofi F, Abbate R, Gensini GF, et al. (2010) Accruing evidence on benefits of adherence to the Mediterranean diet on health: an updated systematic review and meta-analysis. Am J Clin Nutr 92, 1189-1196.

35. Sofi F, Macchi C, Abbate R, et al. (2013) Mediterranean diet and health status: an updated meta-analysis and a proposal for a literature-based adherence score. Public Health Nutr 17, 2769-2782.

36. Dai J, Jones DP, Goldberg J, et al. (2008) Association between adherence to the Mediterranean diet and oxidative stress. Am J Clin Nutr 88, 1364-1370.

37. Kastorini CM, Milionis HJ, Esposito K, et al. (2011) The effect of Mediterranean diet on metabolic syndrome and its components: a meta-analysis of 50 studies and 534,906 individuals. J Am Coll Cardiol 57, 1299-1313.

38. Hu FB (2002) Dietary pattern analysis: a new direction in nutritional epidemiology. Curr Opin Lipidol 13, 3-9.

39. Jacques PF \& Tucker KL (2001) Are dietary patterns useful for understanding the role of diet in chronic disease? $A m \mathrm{~J}$ Clin Nutr 73, 1-2.

40. Samitz G, Egger M \& Zwahlen M (2011) Domains of physical activity and all-cause mortality: systematic review and doseresponse meta-analysis of cohort studies. Int J Epidemiol 40, $1382-1400$

41. Woodcock J, Franco OH, Orsini N, et al. (2011) Non-vigorous physical activity and all-cause mortality: systematic review and meta-analysis of cohort studies. Int J Epidemiol 40, 121-138.
42. Gill JM \& Malkova D (2006) Physical activity, fitness and cardiovascular disease risk in adults: interactions with insulin resistance and obesity. Clin Sci (Lond) 110, 409-425.

43. Ahmed HM, Blaha MJ, Nasir K, et al. (2012) Effects of physical activity on cardiovascular disease. Am J Cardiol 109, 288-295.

44. Radak Z, Hart N, Sarga L, et al. (2010) Exercise plays a preventive role against Alzheimer's disease. J Alzheimers Dis 20, 777-783.

45. Zheng W, McLerran DF, Rolland BA, et al. (2014) Burden of total and cause-specific mortality related to tobacco smoking among adults aged $\geq 45$ years in Asia: a pooled analysis of 21 cohorts. PLOS Med 11, e1001631.

46. U.S. Department of Health and Human Services (2010) How Tobacco Smoke Causes Disease: The Biology and Behavioral Basis for Smoking-Attributable Disease: A Report of the Surgeon General. Atlanta, GA: U.S. Department of Health and Human Services, Centers for Disease Control and Prevention, National Center for Chronic Disease Prevention and Health Promotion, Office on Smoking and Health.

47. WHO (2002) The World Health Report 2002: Reducing Risks, Promoting Healthy Life. Geneva: WHO.

48. van den Brandt PA (2011) The impact of a Mediterranean diet and healthy lifestyle on premature mortality in men and women. Am J Clin Nutr 94, 913-920.

49. Alles B, Samieri C, Feart C, et al. (2012) Dietary patterns: a novel approach to examine the link between nutrition and cognitive function in older individuals. Nutr Res Rev 25, 207-222. 